\title{
Cadaveric study of variations of origin of lateral circumflex and medial circumflex artery in a Sri Lankan Population
}

\author{
Dassanayake $\mathrm{DMTD}^{1}$, Chrishan $\mathrm{AN}^{1}$, Dissanayake $\mathrm{JK}^{1}$ \\ ${ }^{\prime}$ Department of Anatomy, Faculty of Medicine, University of Peradeniya. \\ *thilinidilhara92@gmail.com
}

Lateral circumflex artery (LCA) and medial circumflex artery (MCA) are two main branches of Profunda Femoris artery (PFA) given off on its lateral and medial aspect, respectively. Knowledge on its possible variations is important in preventing iatrogenic injury during surgeries in the hip and bypass surgeries. Twenty seven formalin fixed pre dissected cadavers from the Department of Anatomy, Faculty of Medicine, University of Peradeniya were used. Further dissection was done to expose the site of origin of LCA and MCA. The pattern of origin of the arteries was observed. In cadavers where the MCA and LCA were originating from the Femoral Artery (FA), the distance from the inguinal ligament to the origin of their respective branch was measured using a tape and a string. Eighty three per cent of LCA and $96.2 \%$ of MCA were originating from the PFA but $16.9 \%$ of LCA and $3.7 \%$ of MCA were originating directly from the FA, above the origin of PFA at distances from the inguinal ligament varying from $1.6 \mathrm{~cm}$ $4.2 \mathrm{~cm}$. Two limbs showed a high origin of LCA from FA within $2 \mathrm{~cm}$ from the inguinal ligament. LCA had a common origin with PFA in two cadavers, where the MCA had a normal origin. LCA also had a common origin with MCA from femoral artery in 1 cadaver. This study revealed considerable variations where $16.9 \%$ of LCA and $3.7 \%$ of MCA originated from the FA. This knowledge on variations is important in various surgical and radiological interventions in the femoral triangle. 